\title{
Antibodies and Antimatter: The Resurgence of Immuno-PET
}

The completion of the human genome, coupled with parallel major research efforts in proteomics and systems biology, has led to a flood of information on the roles of individual genes and proteins in normal physiologic processes and their disruptions in disease. In practical terms, this information has opened the door to increasingly targeted therapies as specific molecular markers are identified and validated. The ongoing transition from empiric to molecular medicine has engendered a need for corresponding molecular diagnostics, including noninvasive molecular imaging. Convergence of knowledge regarding key biomarkers that define normal biologic processes and disease with protein and imaging technology makes this an opportune time to revisit the combination of antibodies and PET, or immunoPET.

Key Words: molecular imaging; PET; radioimmunoimaging; antibodies; biomarker imaging; immunoPET

J Nucl Med 2009; 50:2-5

DOI: 10.2967/jnumed.108.056887

Antibodies offer the promise of highly selective binding and recognition of molecular targets and have propelled the identification and analysis of specific proteins in research and in vitro diagnostic laboratories. However, the development of antibodies as clinical imaging agents has had a checkered history, with a handful of antibody tracers for nuclear medicine imaging gaining approval in the 1990s (satumomab pendetide [Oncoscint; Cytogen Corp.], capromab pendetide [Prostascint; Cytogen Corp.], and arcitumomab [CEA-Scan; Immunomedics]). As a group, antibody-based imaging agents have yet to make a significant impact in the clinical or commercial setting (1).

\section{ANTIBODIES-EXPANDING APPLICATIONS IN IMAGING}

Several recent developments are contributing to renewed interest in antibodies as molecular imaging agents (2). As a class, antibodies offer unmatched utility in their ability to recognize essentially any target of interest. In contrast, small molecules and peptides often require the presence of an active site, pocket, or groove for high-affinity interaction. One potential limitation of antibody-based molecular imaging is that the approach is re-

Received Aug. 12, 2008; revision accepted Oct. 21, 2008.

For correspondence or reprints contact: Anna M. Wu, Department of Molecular and Medical Pharmacology, Crump Institute for Molecular Imaging, David Geffen School of Medicine at UCLA, 700 Westwood Plaza, Los Angeles, CA 90095-1770.

E-mail: awu@mednet.ucla.edu

COPYRIGHT @ 2009 by the Society of Nuclear Medicine, Inc. stricted to the assessment of cell surface markers; intracellular targets are not accessible. Nonetheless, the surface-expression patterns of receptors, ligands, adhesion molecules, proteases, and differentiation and activation markers provide a rich source of information on the state of a cell; these proteins represent potential targets for antibody-based molecular imaging (Fig. 1).

Antibodies directed against cell surface targets have already had a significant impact in oncology, cardiology, and immunology in the therapeutic realm. The success of drugs such as trastuzmab (anti-Her2/neu) (Herceptin; Genentech), rituximab (anti-CD20) (Rituxan; Genentech, Inc., and Biogen Idec), bevacizumab (antiVEGF) (Avastin; Genentech), abciximab (antiplatelet IIa/IIIb) (Reo-Pro; Systemic), and adalimumab (anti-TNF- $\alpha$ ) (Humira; Abbott Laboratories), among others, attests to the ability of antibodies to localize and engage or block critical biologic targets in patients. Although antibodies in general are incapable of crossing the blood-brain barrier, antibody therapeutics are finding clinical applications in neurologic diseases such as multiple sclerosis and Alzheimer disease, and strategies for direct delivery to the brain are under development (3). The list of antibody therapeutics moving through phase II/III studies continues to expand, and most major pharmaceutical companies have active antibody development programs. Given the increasing acceptance of antibodies as an effective class of molecular therapeutics, it makes sense to revisit antibodies as potential molecular imaging agents as well. Broad progress in biomarker identification and validation, coupled with advances on both the antibody and antimatter sides, makes immuno-PET increasingly practical and achievable.

\section{SOURCES OF ANTIBODIES FOR MOLECULAR IMAGING}

Current antibody technologies provide robust methods for isolation of novel antibodies specific for any target of interest. First-generation antibody imaging agents were derived from immunogenic mouse monoclonal antibodies. Since then, humanization of murine antibodies for clinical use has become routine, and fully human antibodies can be produced by immunization of mice genetically engineered to carry germline human immunoglobulin genes. Display technologies (phage display, yeast display, ribosome display, etc.) provide an alternative approach for identifying human antibodies with desired binding properties. Recent regulatory approvals of adalimumab and panitumumab (Vectibix; Amgen) validate phage display and transgenic mice as sources of novel human antibodies for clinical use.

Beyond antibodies, interest in alternative small-protein scaffolds as a source of binding molecules for molecular targeting is rapidly expanding (4). Recent examples include single-domain antibodies (of human, camellid, or shark origin), small proteins based on fibronectin domains, ankyrin repeats, or protein A domains (Affibodies; Affibody AB). Alternative scaffolds have been developed to provide binding domains with differing sizes and 
Intracellular targets
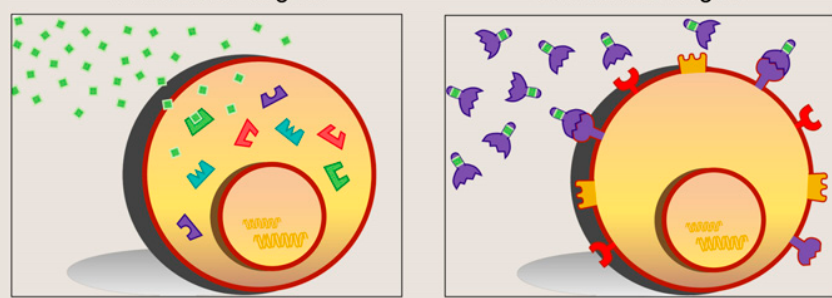

FIGURE 1. Molecular imaging targets include intracellular and cell surface proteins. Antibodies and engineered fragments are particularly suited for detection of cell surface biomarkers (right), which can include highly informative proteins such as growth factor receptors, ligands, adhesion molecules, proteases, and differentiation and activation markers.

shapes, compared with the large, relatively flat binding surfaces of antibodies. Protein domains have been selected for ease of expression in microbial systems and high stability under physiologic and nonphysiologic conditions. Display technology facilitates rapid selection of binding agents with the desired specificity. For example, a monovalent affibody with high affinity for Her2 has been produced and demonstrates excellent in vivo targeting and imaging in tumor-bearing mice $(5,6)$.

\section{ENGINEERED ANTIBODY FRAGMENTS FOR OPTIMIZING PHARMACOKINETICS}

Radiolabeled intact antibodies, with blood-clearance half-lives of days to weeks, are far from optimal for imaging purposes; circulating activity in the blood necessitates long delays between administration of the tracer and acquisition of the final image. Initially, enzymatic digestion of antibodies to yield $\mathrm{Fab}$ or $\mathrm{F}\left(\mathrm{ab}^{\prime}\right)_{2}$ fragments provided a simple approach to reducing background, because fragments lacking the immunoglobulin fragment crystallizable $(\mathrm{Fc})$ region are cleared much more rapidly from the blood. Arcitumomab represents an example of a carcinoembryonic antigen (CEA)-targeting Fab fragment that demonstrated utility in imaging metastatic colon cancer in patients.

The development of antibodies for molecular imaging has been promoted by the development of engineered fragments with pharmacokinetics optimized for imaging (Fig. 2). Reduction of the overall size of antibody-based fragments can result in accelerated blood clearance due to removal of the Fc region (which interacts with neonatal $\mathrm{Fc}$ receptors in vivo to prolong half-life) and by reduction of the molecular weight to a size below the threshhold

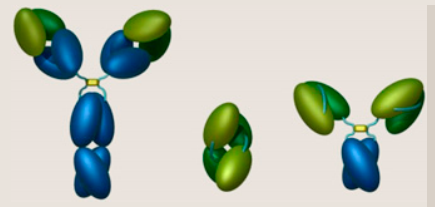

FIGURE 2. Intact antibodies (left) are starting point for immuno-PET tracers. Variable regions, which determine specificity of antibody, are shown in green; constant regions are blue. Diabod-

ies, or dimers of scFv binding domains (center), and minibodies, which are fusions of scFv fragments and antibody constant domain 3 (right), represent examples of engineered antibody fragments with optimal properties for imaging and immuno-PET. for first-pass renal clearance $(\sim 60 \mathrm{kDa})$. Single-chain variable fragments (scFvs) provide a convenient building block for engineered antibody fragments that retain full binding specificity (e.g., diabodies, triabodies, and tetrabodies [dimers, trimers, and multimers of scFv] and tandem diabodies). Fusion of scFvs to additional antibody domains such as the $\mathrm{C}_{\mathrm{H}} 3$ domain to generate minibodies $\left(\mathrm{scFv}_{\mathrm{V}} \mathrm{C}_{\mathrm{H}} 3\right)$ and the closely related small immunoproteins and the larger $\mathrm{scFv}-\mathrm{Fc}$ fusion proteins (7) is another example. These fragments typically retain the high affinity and specificity of the parental antibody but also exhibit a range of blood-clearance properties that are format-dependent (8).

Successful imaging requires that the tracer must circulate long enough to allow sufficient perfusion and binding in target tissues, coupled with reasonably fast clearance from the circulation. Diabodies have been developed in a variety of antibody or antigen systems and exhibit terminal half-lives in murine models in the range of 3-5 h. Cancer-specific diabodies can reach levels of $7 \%-10 \%$ injected dose per gram in tumor xenograft models. Retention in target tissue is excellent, because of the bivalent binding of these fragments, and high-contrast small-animal PET images can be obtained within a few hours after tracer injection $(9,10)$. Bivalent minibodies above the threshhold for renal clearance exhibit intermediate kinetics. As a result, higher overall uptake levels ( $\sim 20 \%$ injected dose/g) can be achieved in target tissues, resulting in excellent imaging, although optimal imaging times may be longer. High-affinity, monovalent Her2-specific affibodies demonstrated impressive targeting and retention in preclinical models (5), counter to the prevailing wisdom that bivalent molecules are necessary for optimal targeting and retention.

\section{Clinical Potential}

A high priority is translation of engineered antibody-based imaging agents to establish pharmacokinetics and targeting in patients, because prediction of clinical performance based on preclinical studies in rodents is challenging. Initial SPECT studies using ${ }^{123}$ I-labeled L19 $(\mathrm{scFv})_{2}$ specific for fibronectin ED-B (a biomarker of neovascularization) showed that detection of tumorassociated angiogenesis in patients was feasible at $6 \mathrm{~h}$ after injection (11). A subsequent phase I/II clinical study similarly showed excellent targeting in 4 of 5 patients with head and neck squamous cell carcinoma (12). Planar and SPECT studies using the ${ }^{123} \mathrm{I}$-cT84.66 CEA-specific minibody in a presurgical study of patients with recurrent colorectal carcinoma demonstrated localization to 8 of 10 lesions larger than $1.0 \mathrm{~cm}$ (13).

Encouraging results from these and other proof-of-principle studies using engineered fragments labeled with single photonemitting isotopes lay a strong foundation for the development of these reagents as immuno-PET tracers, given the higher sensitivity and resolution of PET scanners and the ability to quantitate activity—and thus expression levels—of molecular targets in vivo.

\section{ANTIMATTER-PROGRESS WITH POSITRON-EMITTING RADIONUCLIDES}

\section{Matching Biologic and Physical Half-Lives}

Marrying antibodies and positron-emitting radionuclides for PET applications requires an appropriate match between the biologic half-life of the protein and the physical half-life of the isotope. For example, it makes little sense to radiolabel an intact antibody (half-life $\left[\mathrm{t}_{1 / 2 \beta}\right]=7-14 \mathrm{~d}$ ) with ${ }^{18} \mathrm{~F}\left(\mathrm{t}_{1 / 2}=109 \mathrm{~min}\right)$; by the time optimal antibody targeting, distribution, and clearance 
are achieved in vivo, any potential signal will have decayed. Successful implementation of immuno-PET requires access to radionuclides with a longer half-life. For this reason, initial encouraging demonstrations of immuno-PET in the 1990s remained limited in their effect because of the lack of broad access to "exotic" PET radionuclides such as ${ }^{124} \mathrm{I}$ and ${ }^{64} \mathrm{Cu}$. More recently, with the widespread adoption of PET using ${ }^{18} \mathrm{~F}$-FDG, PET scanners have become ubiquitous in nuclear medicine departments, and attention has increasingly turned to the development of novel molecular tracers for use in PET.

Several PET radionuclides have been the focus of recent clinical and preclinical studies (Table 1). Ideally, alignment of the physical half-life of the radiolabel and biologic half-life of the tracer will lead to optimal imaging performance. In practical terms, combinations that may appear suboptimal (such as radiolabeling small, rapid-clearing fragments with ${ }^{124} \mathrm{I}$ (9) or intact antibodies with ${ }^{64} \mathrm{Cu}(14,15)$ or $\left.{ }^{86} \mathrm{Y}(16)\right)$ have nonetheless yielded highly informative images and data in preclinical studies.

Several additional considerations must be weighed in selecting the appropriate label $(1,17)$. In addition to half-life, the positron yield and existence of concomitant emissions $\left(\beta^{-}, \gamma\right)$ will have major effects on the amount of activity that needs to be administered and the radiation dose to the patient or health care providers. Positron range may affect resolution if the positron travels a significant distance before annihilation. Finally, commercial availability and cost of positron-emitting radionuclides are also significant issues that continue to shape (or impede) progress in the development of immuno-PET.

PET radionuclides with half-lives on the order of days would be ideally suited for conjugation with intact antibodies for clinical imaging (Table 1), and recent efforts have gravitated toward ${ }^{89} \mathrm{Zr}$ $(78.4 \mathrm{~h})$ and ${ }^{124} \mathrm{I}(100.2 \mathrm{~h})$. Recent examples of radiolabeling and preclinical evaluation of intact antibodies with ${ }^{89} \mathrm{Zr}$ include labeling of ibritumomab tiuxetan (Zevalin; Cell Therapeutics Inc.) with ${ }^{89} \mathrm{Zr}$ (18) and development of a ${ }^{89} \mathrm{Zr}$-Met-specific antibody (19). The clinical utility of ${ }^{89} \mathrm{Zr}$-labeled U36, a chimeric antibody specific for CD44v6, has been demonstrated in a series of 20 patients with head and neck cancer (20). All of the primary tumors and $72 \%$ of the lymph node metastases (confirmed by pathology) were detected in this clinical immuno-PET study. Improved commercial availability of ${ }^{124} \mathrm{I}$ has also led to renewed interest in clinical evaluation. Immuno-PET imaging of 26 patients with renal masses after injection of ${ }^{124} \mathrm{I}-\mathrm{cG} 250$ (anticarbonic anhydrase-IX) resulted in successful detection of 15 of 16 clear-cell carcinomas (21). In these presurgical studies, the final immuno-PET scans were acquired 6-8 d after injection, just before surgery.

\begin{tabular}{|c|c|c|}
\hline Radionuclide & Half-life & Positron yield \\
\hline${ }^{68} \mathrm{Ga}$ & $68 \mathrm{~min}$ & $89 \%$ \\
\hline${ }^{18} \mathrm{~F}$ & $109 \mathrm{~min}$ & $97 \%$ \\
\hline${ }^{64} \mathrm{Cu}$ & $12.7 \mathrm{~h}$ & $18 \%$ \\
\hline${ }^{86} Y$ & $14.7 \mathrm{~h}$ & $17.5 \%$ \\
\hline${ }^{76} \mathrm{Br}$ & $16.0 \mathrm{~h}$ & $55 \%$ \\
\hline${ }^{89} \mathrm{Zr}$ & 78.4 & $22.7 \%$ \\
\hline $124 \mid$ & $100.2 \mathrm{~h}$ & $23 \%$ \\
\hline
\end{tabular}

Data from Interactive Chart of Nuclides at www.nndc.bnl.gov/ nudat2 and (16).
Antibody fragments offer practical advantages for immuno-PET applications, because they can reduce the interval between injection and imaging to hours instead of the days required for clearance of intact antibodies. PET radionuclides with short or intermediate halflives (Table 1) have been used in combination with fragments, engineered and conventional, in a growing body of preclinical studies. Conventional $\mathrm{F}\left(\mathrm{ab}^{\prime}\right)_{2}$ fragments derived from trastuzumab and radiolabeled with ${ }^{68} \mathrm{Ga}$ have been used to evaluate response to treatment by in vivo PET of Her2 expression after administration of 17-AAG, a drug that accelerates Her2 degradation (22).

Immuno-PET detection of Her2 expression in tumor xenografts in mice has also been achieved using ${ }^{124} \mathrm{I}-\mathrm{C} 6.5$ diabodies (10), ${ }^{64} \mathrm{Cu}$-labeled minibodies, and $\mathrm{scFv}-\mathrm{Fc}$ fragments derived from trastuzumab (23). ${ }^{76} \mathrm{Br}$-labeled L19 small-immunoprotein fragments were successfully used to image tumor neovasculature in murine models, although normal-tissue uptake was observed in the ovaries and uterus, and partial debromination was observed (24). Pretargeted imaging using a multivalent, recombinant bispecific antibody has been transitioned to PET detection by using a ${ }^{124}$ I-radiolabeled hapten peptide as the tracer (25). One of the most promising developments, with implications for clinical translation, is the demonstration of rapid ${ }^{18} \mathrm{~F}$ labeling and imaging of colon carcinoma xenografts using an anti-CEA diabody (26). The antigen-positive tumor was readily detected by small-animal PET imaging at time points from 1 to $6 \mathrm{~h}$ after injection.

Finally, realization of protein-based PET tracers will require continued innovations in conjugation and radiolabeling methods. As various cell surface proteins are proposed as candidates for antibody imaging, an important first question is whether the antibody or antigen complex is internalized. If not, then simple radiohalogenation methods can be used to generate a suitable tracer, and the radioactive signal will persist at the target site. Figure 3 shows that minibodies that recognize the noninternalizing targets CEA and prostate stem cell antigen (glycosylphosphatidylinositol-linked membrane proteins) or CD20 (integral membrane protein) provide excellent small-animal PET images after standard
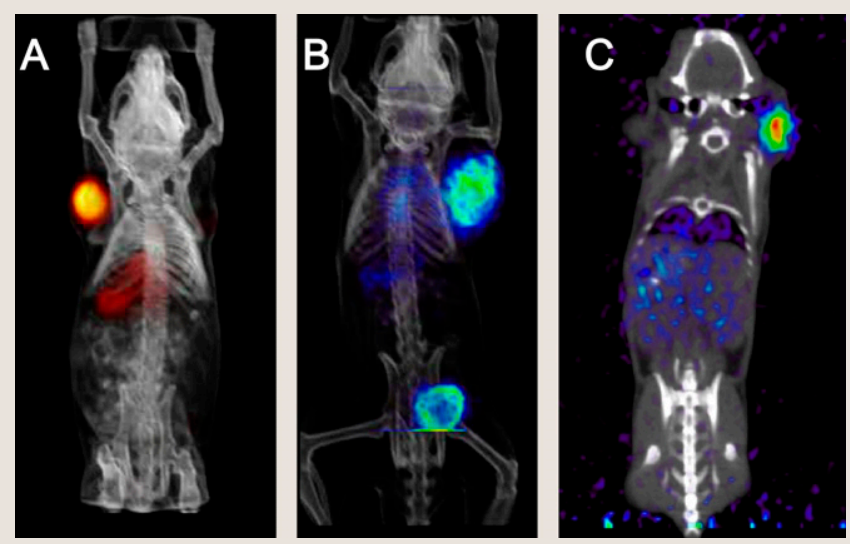

FIGURE 3. ${ }^{124}$ I-labeled minibodies demonstrate excellent small-animal PET imaging of tumor xenografts expressing noninternalizing targets such as CEA (A), prostate stem cell antigen (B), and CD20 (C), at $20 \mathrm{~h}$ after intravenous injection. Nontargeted minibody $(80 \mathrm{kDa})$ clears via liver (visible in $\mathrm{A}$ and $\mathrm{C}$ ); catabolism releases iodide and iodotyrosine, which are rapidly excreted via kidneys and bladder (visible in B). Imaging of internalizing targets requires conjugation with residualizing radiolabel. 
radioiodination with ${ }^{124} \mathrm{I}$. An added advantage is that a nonlocalized tracer is cleared to the liver, kidney, or spleen and catabolized with rapid clearance of the radioactive metabolites. As a result, normal-tissue background is low. However, many cell surface targets of interest are internalized, necessitating the use of a residualizing radiolabel. Stabilized iodination protocols (10), or radiometal chelates, are required. Continued development of improved bifunctional chelates for positron-emitting radionuclides continues to be of critical importance.

\section{CONCLUSION}

An impressive range of positron-emitting radionuclides (Table 1) and a broad spectrum of molecular targets are under active investigation in immuno-PET studies. Progress is accelerating because of the identification of key cell surface markers as potential molecular imaging biomarkers, broad antibody technology that facilitates rapid isolation and reformatting into immuno-PET tracers, and advances in production and use of additional PET radionuclides for labeling biomolecules. In oncologic applications, availability of PET tracers for assessment of cell surface biomarkers can play an important role at many points along the cancer-care continuum: for tumor detection and identification (i.e., tissue of origin); at diagnosis, staging, and restaging; for treatment selection and monitoring; and during follow-up. Immuno-PET can potentially be used to evaluate target expression and accessibility before treatment with an antibody therapeutic. Quantitation of tissue uptake and dosimetry for radioimmunotherapy is an area to which immuno-PET can contribute significantly, because of the availability of matched imaging or therapeutic radioisotope pairs, such as ${ }^{64} \mathrm{Cu} /{ }^{67} \mathrm{Cu},{ }^{86} \mathrm{Y} /{ }^{90} \mathrm{Y}$, and ${ }^{124} \mathrm{I} /{ }^{131} \mathrm{I}$. Challenges remain, because many targets that are efficacious in therapeutic applications (such as epidermal growth factor receptor) will not be imageable because of normal-tissue expression. Radionuclide availability and continued development of radiochemistry and coordination chemistry are areas that need to be addressed if broad clinical use is envisioned. Nonetheless, immuno-PET will provide a means to "fingerprint" the cell surface and tissues in vivo, and translation to the clinical setting will add a new dimension to our understanding of disease and its treatment.

Anna M. Wu
David Geffen School of Medicine at UCLA
Los Angeles, California

\section{REFERENCES}

1. Boswell CA, Brechbiel MW. Development of radioimmunotherapeutic and diagnostic antibodies: an inside-out view. Nucl Med Biol. 2007;34:757-778.

2. Wu AM, Olafsen T. Antibodies for molecular imaging of cancer. Cancer J. 2008;14:191-197.

3. Pardridge WM. Re-engineering biopharmaceuticals for delivery to brain with molecular Trojan horses. Bioconjug Chem. 2008;19:1327-1338.

4. Nuttall SD, Walsh RB. Display scaffolds: protein engineering for novel therapeutics. Curr Opin Pharmacol. July 19, 2008 [Epub ahead of print].

5. Orlova A, Magnusson M, Eriksson TL, et al. Tumor imaging using a picomolar affinity HER2 binding affibody molecule. Cancer Res. 2006;66:4339-4348.
6. Tolmachev V, Orlova A, Nilsson FY, Feldwisch J, Wennborg A, Abrahmsén L. Affibody molecules: potential for in vivo imaging of molecular targets for cancer therapy. Expert Opin Biol Ther. 2007;7:555-568.

7. Holliger P, Hudson PJ. Engineered antibody fragments and the rise of single domains. Nat Biotechnol. 2005;23:1126-1136.

8. Kenanova V, Olafsen T, Crow DM, et al. Tailoring the pharmacokinetics and positron emission tomography imaging properties of anti-carcinoembryonic antigen single-chain Fv-Fc antibody fragments. Cancer Res. 2005;65:622-631.

9. Sundaresan G, Yazaki PJ, Shively JE, et al. ${ }^{124}$ I-labeled engineered anti-CEA minibodies and diabodies allow high-contrast, antigen-specific small-animal PET imaging of xenografts in athymic mice. J Nucl Med. 2003;44:1962-1969.

10. Robinson MK, Doss M, Shaller C, et al. Quantitative immuno-positron emission tomography imaging of HER2-positive tumor xenografts with an iodine-124 labeled anti-HER2 diabody. Cancer Res. 2005;65:1471-1478.

11. Santimaria M, Moscatelli G, Viale GL, et al. Immunoscintigraphic detection of the ED-B domain of fibronectin, a marker of angiogenesis, in patients with cancer. Clin Cancer Res. 2003;9:571-579.

12. Birchler MT, Thuerl C, Schmid D, et al. Immunoscintigraphy of patients with head and neck carcinomas, with an anti-angiogenetic antibody fragment. Otolaryngol Head Neck Surg. 2007;136:543-548.

13. Wong JY, et al. Pilot trial evaluating an ${ }^{123} \mathrm{I}$-labeled 80 -kilodalton engineered anticarcinoembryonic antigen antibody fragment (cT84.66 minibody) in patients with colorectal cancer. Clin Cancer Res. 2004;10:5014-5021.

14. Ping Li W, Meyer LA, Capretto DA, Sherman CD, Anderson CJ. Receptorbinding, biodistribution, and metabolism studies of ${ }^{64} \mathrm{Cu}$-DOTA-cetuximab, a PET-imaging agent for epidermal growth-factor receptor-positive tumors. Cancer Biother Radiopharm. 2008;23:158-171.

15. Cai W, Wu Y, Chen K, Cao Q, Tice DA, Chen X. In vitro and in vivo characterization of ${ }^{64} \mathrm{Cu}$-labeled Abegrin, a humanized monoclonal antibody against integrin $\alpha_{\mathrm{v}} \beta_{3}$. Cancer Res. 2006;66:9673-9681.

16. Parry R, Schneider D, Hudson D, et al. Identification of a novel prostate tumor target, mindin/RG-1, for antibody-based radiotherapy of prostate cancer. Cancer Res. 2005;65:8397-8405.

17. van Dongen GA, Visser GW, Lub-de Hooge MN, de Vries EG, Perk LR. Immuno-PET: a navigator in monoclonal antibody development and applications. Oncologist. 2007;12:1379-1389.

18. Perk LR, Visser OJ, Stigter-van Walsum M, et al. Preparation and evaluation of ${ }^{89} \mathrm{Zr}$-Zevalin for monitoring of ${ }^{90} \mathrm{Y}$-Zevalin biodistribution with positron emission tomography. Eur J Nucl Med Mol Imaging. 2006;33:1337-1345.

19. Perk, LR, Stigter-van Walsum M, Visser GWM, et al. Quantitative PET imaging of Met-expressing human cancer xenografts with ${ }^{89} \mathrm{Zr}$-labelled monoclonal antibody DN30. Eur J Nucl Med Mol Imaging, 2008;35:1857-1867.

20. Börjesson PK, Jauw YW, Boellaard R, et al. Performance of immuno-positron emission tomography with zirconium-89-labeled chimeric monoclonal antibody U36 in the detection of lymph node metastases in head and neck cancer patients. Clin Cancer Res. 2006;12:2133-2140.

21. Divgi CR, Pandit-Taskar N, Jungbluth AA, et al. Preoperative characterisation of clear-cell renal carcinoma using iodine-124-labelled antibody chimeric G250 $\left({ }^{124} \mathrm{I}-\mathrm{cG} 250\right)$ and PET in patients with renal masses: a phase I trial. Lancet Oncol. 2007;8:304-310.

22. Smith-Jones PM, Solit D, Afroze F, Rosen N, Larson SM. Early tumor response to Hsp90 therapy using HER2 PET: comparison with ${ }^{18} \mathrm{~F}$-FDG PET. J Nucl Med. 2006;47:793-796

23. Olafsen T, Kenanova VE, Sundaresan G, et al. Optimizing radiolabeled engineered anti-p185HER2 antibody fragments for in vivo imaging. Cancer Res. 2005;65:5907-5916.

24. Rossin R, Berndorff D, Friebe M, Dinkelborg LM, Welch MJ. Small-animal PET of tumor angiogenesis using a ${ }^{76} \mathrm{Br}$-labeled human recombinant antibody fragment to the ED-B domain of fibronectin. J Nucl Med. 2007;48:1172-1179.

25. Sharkey RM, Karacay H, McBride WJ, Rossi EA, Chang CH, Goldenberg DM. Bispecific antibody pretargeting of radionuclides for immuno single-photon emission computed tomography and immuno positron emission tomography molecular imaging: an update. Clin Cancer Res. 2007;13(18 suppl):5577S$5585 \mathrm{~S}$.

26. Cai W, Olafsen T, Zhang X, et al. PET imaging of colorectal cancer in xenograftbearing mice by use of an ${ }^{18} \mathrm{~F}$-labeled $\mathrm{T} 84.66$ anti-carcinoembryonic antigen diabody. J Nucl Med. 2007;48:304-310. 